Fecha de recepción: febrero 2019

Fecha de aceptación: julio 2019

Versión final: octubre 2020

\section{Epistemología y metodología en y de la investigación en Diseño}

Roxana Ynoub *

Resumen: El objetivo de este escrito es el de aportar elementos de juicio al debate en torno a la epistemología y la metodología de investigación en el campo del Diseño. En esa dirección se comienza por definir el alcance de la epistemología como disciplina que se ocupa de la reflexión sobre las condiciones de producción y validación del conocimiento científico. A partir de ello se revisan los rasgos distintivos del conocimiento en el campo del Diseño, situándolo como conocimiento proyectual. Se propone que dichos rasgos distintivos comprometen asuntos que han sido problemáticos para la epistemología clásica: en particular porque vinculan cuestiones factuales ("lo que es") con cuestiones valorativas ("lo que debe ser" o sobre "lo deseable"). Conforme con la tradición positivista, de enunciados factuales no se pueden derivar enunciados modales o valorativos. Se propone entonces asumir el examen del Diseño como una praxis, es decir, como una práctica social, para desde allí revisar sus fundamentos epistemológicos. Se postula que esta perspectiva permite superar el supuesto antagonismo entre el orden factual y valorativo, integrando el abordaje desde la complejidad de la experiencia, vital y social en que la práctica del Diseño se realiza. Desde esta definición se ubican las diversas dimensiones analíticas con las que la referida complejidad puede ser abordada, ubicando para cada una de ellas especificidades temáticas y disciplinarias. Finalmente se examinan las consecuencias que este modelo tiene para tematizar la investigación científica en el campo del Diseño.

Palabras clave: epistemología - metodología de la investigación - Diseño - praxis.

[Resúmenes en inglés y portugués en la página 31]

${ }^{*}$ Doctora en Psicología (2002) y Lic. en Psicología (1990) por la Universidad de Buenos Aires. En 1995 recibe el Premio a la contribución científica técnica de la misma universidad. Pertenece a distintas Sociedades Científicas del país y a nivel regional. Posee una amplia experiencia de investigación en proyectos nacionales e internacionales y ha realizado actividades de Consultoría y Asesoramiento para distintos organismos nacionales. Desempeña desde 1992 tareas docentes en carreras de grado y como profesora titular y adjunta en posgrados de la Universidad de Buenos Aires y de diversas Universidades Nacionales (de Lanús, Lomas de Zamora, Mar del Plata, Rosario, La Plata, La Pampa y Entre Ríos), y privadas (Universidad de Morón, UCES, etc.). 


\section{La cuestión epistemológica}

Se propone en este escrito aportar algunas reflexiones al debate -actualmente muy prolífico y activo- sobre el puesto de la epistemología y la metodología en el campo del Diseño ${ }^{1}$. En esa dirección, el primer paso será ofrecer una definición de epistemología para, a partir de ella, dar marco a la revisión propuesta.

Resulta de interés comenzar por recordar entonces, que la epistemología constituye un sub-dominio del campo filosófico.

La filosofía es una disciplina reflexiva. Su objeto de análisis es el pensamiento. Pero no se ocupa del pensamiento como podría hacerlo por ejemplo la psicología, considerando los aspectos cognitivos o emotivos vinculados a él. Se ocupa del "pensamiento que piensa o examina el pensamiento". De suerte que la filosofía puede llamarse "pensamiento en segundo grado" (cfr. Collingwood, 1977). Así por ejemplo, a la filosofía no le interesa averiguar si cierto objeto es o no es bello, si cierta ley es o no es justa, si cierto conocimiento es o no es verdadero. Se interesa más bien por averiguar qué es «lo bello», qué es «la justicia», qué es «la verdad». Y lo hace, como se dijo, por vía reflexiva, examinando las categorías con las que pensamos lo bello, lo justo, lo verdadero; o las maneras por las que adscribimos belleza a un objeto, reconocemos la justicia, o admitimos la verdad de un conocimiento. Las distintas ramas de la filosofía se ocupan de regiones específicas de este tipo de análisis. Por ejemplo, la lógica se interesa por las formas en que se encadenan o vinculan los pensamientos en el marco de juicios y razonamientos. La metafísica se ocupa de la reflexión sobre nociones fundamentales del pensar, es decir, sobre nociones que están a la base de muchas otras nociones particulares. Por ejemplo, las nociones de «ser», «existencia», «causa», «sustancia», etc. Así, por ejemplo, cada vez que se explica un hecho (sea desde nuestro conocimiento vulgar o científico), se invoca seguramente una causa explicativa-cualquiera sea el asunto considerado-. Sin embargo, al hacerlo no se detienen en la noción misma de «causa». Ella no es el asunto de nuestra explicación, sino un medio para organizarla y hacerla posible. Es allí precisamente en dónde la reflexión filosófica encuentra asuntos de interés: en este caso en el examen de esas mediaciones intelectivas, que no las tenemos delante nuestro como un objeto, porque son en verdad la condición -o el medio necesario- para tener objetos, para representarnos y dar sentido a nuestra experiencia.

La epistemología es también una rama de la filosofía. Atiende a las condiciones de producción y validación de un tipo peculiar de conocimiento, que es el conocimiento científico. El término proviene del vocablo griego episteme ( $\dot{\varepsilon} \pi \sigma \tau \eta \dot{\mu \eta})$ que significa conocimiento fundado o válido, y que se utilizaba en su origen por oposición a la doxa ( $\delta$ ó $\xi \alpha)$ que refiere al conocimiento vulgar e infundado.

Más allá de las múltiples vertientes que hoy coexisten en el escenario epistemológico, todas ellas se definen en relación al examen de las condiciones de posibilidades del conocimiento científico. Se interesan por evaluar el alcance de este conocimiento, los caminos para alcanzarlo, las especificidades que adquieren en distintos dominios disciplinarios, entre otras interesantes y decisivas cuestiones.

Para los fines que se persiguen en este escrito, no resulta necesario (ni materialmente posible) detenernos a indagar aquí las muy distintas maneras en que las epistemologías (en plural) han planteado la cuestión de la fundamentación del conocimiento científico. Tam- 
poco las controversias entre escuelas epistemológicas actualmente en boga. Entre otras cosas porque el tema que nos ocupa abre cuestiones novedosas incluso para los debates tradicionales en el campo epistemológico ${ }^{2}$.

Lo que sí interesa, es situar el puesto específico que las ciencias del Diseño tienen en relación a otras ciencias (que son las usualmente consideradas por la reflexión epistemológica). A partir de ello, se puede aplicar el examen categorial al campo del Diseño, de modo tal de averiguar las características que adquiere la producción de conocimiento en este dominio.

Una distinción consagrada para clasificar los distintos tipos de ciencia, considera:

a. por una parte, a las llamadas ciencias formales,

b. por otra, a las ciencias fácticas o empiricas las que a su turno, las divide en dos grandes grupos:

b.1. ciencias naturales.

b.2. ciencias sociales y humanas.

En lo que respecta a las ciencias formales sus objetos son «objetos ideales», como por ejemplo, lo son los de la lógica y la matemática. Estas disciplinas se interesan por identificar y formalizar el tipo de reglas que operan con estos objetos ideales (por ejemplo, las operaciones aritméticas o las reglas de derivación lógica). Se las llama formales porque no se interesan estrictamente por los contenidos del saber, sino por las formas en que cualquier saber puede organizarse. Se preguntan sobre el carácter necesario de los conocimientos pero examinandos en términos de su validez formal (aunque por las mismas razones pueden interesarse por averiguar las condiciones de falsedad de un conocimiento, de contradicción, incompletud, el absurdo o la contingencia de un saber).

En cambio, las ciencias fácticas, refieren a «objetos empíricos», es decir, cuya materialidad trasciende el orden del pensamiento.

Sin duda las características de estos objetos, sus propiedades y los modos de aproximación que se dan en el marco de unas y otras ciencias, son considerablemente diferentes por un sinnúmero de razones. Sin embargo, puede afirmarse que, en principio, en todos los casos los objetos de estudio se asumen como hechos a priori a su investigación. Así, por ejemplo, la sociología entendida como la disciplina que estudia la sociedad, asume que ese objeto (la sociedad humana) es un hecho del mundo, y que por lo tanto puede ser investigado. En el mismo sentido en que la biología admite como punto de partida, que la vida se manifiesta de múltiples formas, y que todas ellas pueden ser objeto de investigación ${ }^{3}$.

Aunque cada enfoque de investigación construye su objeto como resultado de sus definiciones conceptuales y operacionales, esa construcción parte de aceptar la materialidad de su objeto como un a priori de experiencia.

Por el contrario, en el orden de las ciencias del Diseño, que conforme a la definición de Herbert Simon (973), podemos definir como las ciencias de los objetos construidos, nos encontramos con que su objeto (al menos en términos "fácticos") no constituye un dato a priori a la experiencia del Diseño: ni como materialidad objetiva, ni como producción subjetiva (proyecto/idea). El objeto es creado en el acto y por medio de la actividad de Diseño (sea como idea proyectada, sea como objeto construido). 
Una consecuencia de esta importante diferencia, estriba en el alcance y el tipo de conocimiento que insumen y producen cada una de estas ciencias.

Las ciencias fácticas (sea en el orden de los fenómenos sociales o naturales), se interrogan por el ser de los objetos, y estipulan las condiciones metodológicas por medio de las cuales se adquiere o se accede a un saber sobre ellos. De modo tal que su aparato categorial se basa en enunciados factuales (es decir, refieren a lo que las cosas son).

Mientras que en el campo del Diseño, el objeto es resultado de una construcción, de una elaboración producto de la imaginación, y orientada por fines. En términos categoriales atiende a lo que las cosas deben ser, pueden ser o es deseable que sea conforme a dichos fines, fijados por los intereses y perspectivas del Diseño. Fue precisamente Herbert Simon, uno de los primeros en advertir esta especificidad de las ciencias del Diseño:

Histórica y tradicionalmente, ha venido correspondiendo a las disciplinas científicas enseñar cuanto hace referencia a las cosas naturales: cómo son y cómo funcionan. En tanto que correspondía a las escuelas de ingeniería enseñar cuanto hace referencia a las cosas artificiales: cómo hacer artefactos que reuniesen unas determinadas propiedades, cómo diseñar. Diseña todo aquel que concibe unos actos destinados a transformar situaciones existentes en otras, más dentro de su preferencia (Simon, 1978, p. 87).

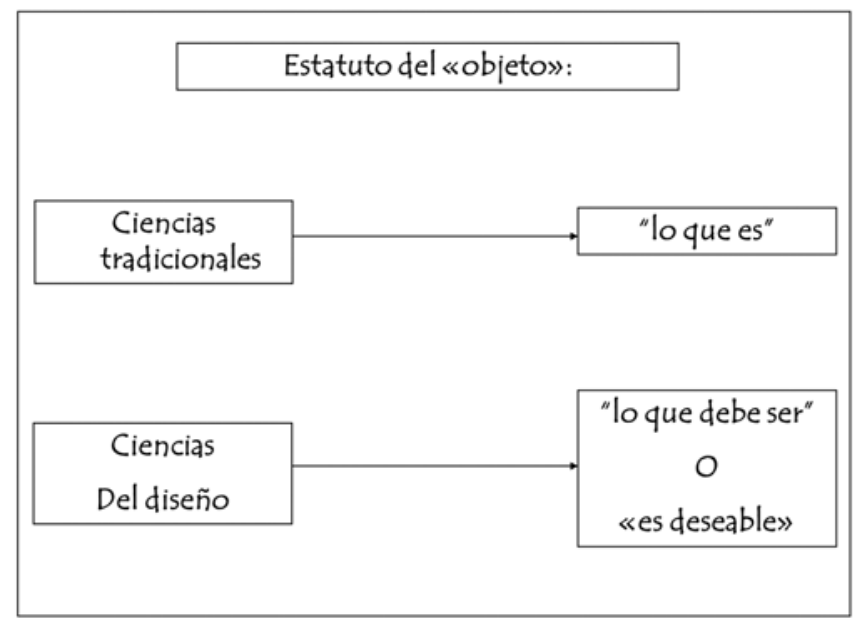

En el orden de las ciencias del Diseño, el objeto refiere precisamente a la actividad o práctica productiva/imaginativa. Se trata de los procesos de producción de un tipo particular de conocimiento, que puede definirse como conocimiento proyectual. 
En torno a estas decisivas cuestiones se ubican los principales desafíos que tiene planteada una epistemología del Diseño.

En particular porque enfrenta el problema de vincular enunciado sobre hechos (orden del ser) a prescripciones sustentadas en valores (deber ser/ desear ser).

David Hume un referente de la filosofía moderna, postuló un límite entre estos dominios. Conforme con él, del ser no se puede derivar el deber ser. O, dicho de otra manera, de enunciados factuales no se pueden derivar enunciados morales o prescriptivos.

Sin embargo, el Diseño como actividad proyectual, no puede concebirse sino es por referencia a fines. Y los fines suponen valores. Como se ha señalado, se valora lo que es a la luz de lo que podría ser, debería ser, o es deseable que sea.

Como ya se señaló, estas son las razones que abren un sinnúmero de consecuencias epistemológicas, lógicas y metodológicas, para la reflexión en el campo del Diseño.

Aunque no se pretende saldar aquí tan complejas cuestiones, en lo que sigue se detallarán en la consideración de algunas de ellas.

\section{El Diseño como táctica y estrategia ${ }^{4}$}

Diseñar supone escoger acciones entre alternativas posibles, para alcanzar cierto fin deseado. Dado que un mismo fin puede alcanzarse por diversos medios, la elección de los medios, compromete la tarea de Diseño. Así, por ejemplo, un objeto para sentarse, puede ser concebido conforme a múltiples criterios:

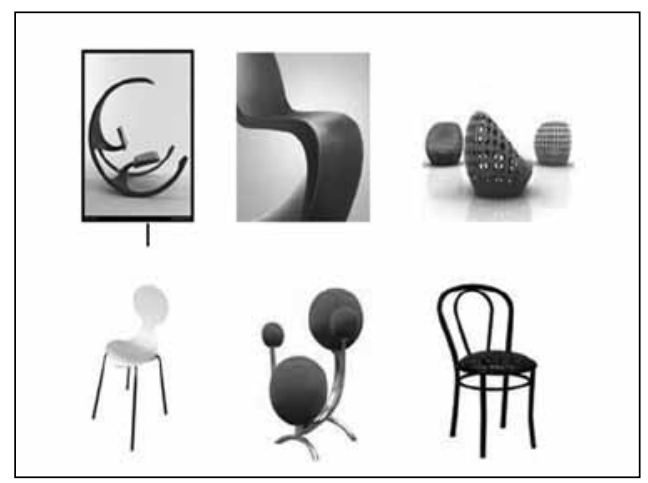

El principio de equifinalidad -acuñado en el marco de la teoría de sistemas- expresa precisamente esta idea: los mismos fines pueden alcanzarse por múltiples medios.

Y esto no ocurre sólo en las ciencias de lo artificial (Simon, 1978). También el orden natural encuentra muy diversos caminos para alcanzar idénticos fines: ¿ide cuántas maneras 
se puede "diseñar" una nariz? Admitiendo que todas ellas cumplen los mismos propósitos (es decir, tienen una función y una posición homóloga en diversos vivientes) la variedad es tan grande como grande es el espectro de especies animales que se sirven de respiración pulmonar.

De modo que, diseñar es una actividad orientada por fines y, en el caso, de los diseños de origen humano, esa actividad supone la elección deliberada de determinados cursos de acción (entre múltiples posibles), con vistas a maximizar determinados valores comprometidos con el logro de dichos fines.

Se podría afirmar que los fines comprometen la estrategia; mientras que los medios para alcanzarlos, las decisiones tácticas.

Así, por ejemplo, si se atiende a lo estético se deberá considerar probablemente el contexto en que va a utilizarse el objeto; si en cambio el valor apunta a lo funcional, habrá que evaluar (al menos en el caso de las sillas) su adecuación ergonómica; si se considera lo económico se tendrá en cuenta el costo; si se valora en términos ecológicos, se considerará el impacto medioambiental de los materiales. En cada uno de estos criterios, se alude a un valor específico: la funcionalidad, el costo, la belleza, la sustentabilidad ambiental, etc. son todos valores. Se desea que el objeto sea funcional, bello, sustentable, etc.

Desde una perspectiva racionalista se podría postular, como un desiderátum, el de maximizar todos los valores (o al menos los valores en que se interesa) conjuntamente.

Sin embargo, y aún cuando se han sugerido métodos científicos y formalizados para ello, esta pretensión no deja de ser un ideal regulativo (para decirlo parafraseando a Inmanuel Kant). En primer lugar porque muchos de los valores comprometidos en el Diseño no pueden ser parametrizados (ni lo estético, ni lo ético, por lo menos). Por otra parte porque, por lo general, la realización de uno de los valores entra en contradicción con la realización de otro u otros (podría suceder, por ejemplo, que en aras de economizar se afecte la estética o el medioambiente, o, también que una decisión a beneficio de la estética, atente contra la funcionalidad).

Si bien estas reflexiones no agregan nada novedoso a lo que usualmente comprende quien diseña (los valores que hemos comentado podrían remitirse a la clásica tríada de Vitrubio $\left.^{6}\right)$, interesan en tanto permiten comprender la estructura lógico-categorial comprometida en la praxis del Diseño. Asunto al que atiende precisamente la epistemología, tal como lo señalamos al comienzo de este escrito.

En tanto ciencias de lo artificial, las ciencias del Diseño comparten su campo con otras tantas disciplinas como la medicina (las ciencias de la salud en general), la ingeniería, el derecho, por citar algunas de ellas. En todos los casos se advierte la misma cuestión que acabamos de examinar: la relación entre el ser y el deber ser. Lo factual (lo que es) examinado a la luz de criterios normativos/valorativos (lo que debe ser o es deseable que sea).

En el terreno de las ciencias de la salud, Canguilhem, G. lo expresó magistralmente al definir la práctica y el objeto de la medicina en términos de normal y patológico: "Sin los conceptos de normal y patológico el pensamiento y la actividad del médico son incomprensibles" (1978, p. 183).

Distinguir entre un estado normal y uno patológico, implica juzgar lo que es a la luz de lo que debiera ser (o se espera). Requiere estipular un valor para establecer la distinción (de 
la misma manera que un hecho es lícito o ilícito en base a una normativa desde la que se lo juzga -cambia la normativa y cambia la licitud de un mismo hecho).

El concepto de patología se aplica también al campo del Diseño, y remite exactamente a la misma idea valorativa: se examina lo que es a la luz de los que debería ser -según valores asumidos.

En este punto se impone entonces la pregunta: ¿es posible una ciencia de lo que debe ser? Ya se ha señalado que para el positivismo clásico la respuesta es negativa. La ciencia-según esa concepción- debía desentenderse de todo juicio valorativo. Entre otras cosas porque el orden de los valores queda, para dicha concepción, emparentado con una posición subjetivista.

$\mathrm{Si}$, pese a ello, se acepta aquí la posibilidad de esta ciencia, se abren entonces otras preguntas: ¿cuál es su objeto? ¿cuál es su método? ¿cuál es la lógica que la rige?

De modo más específico en las ciencias del Diseño, su objeto es

- ¿el proceso de Diseño?

- ¿el producto/artefacto diseñado?

- ¿el proyecto como plan o ideación de Diseño?

Según cómo se defina al objeto de este campo, las consecuencias epistemológicas, ontológicas y metodológicas cambian sensiblemente. Sin embargo, parece sensato considerar que todas esas dimensiones se reclaman unas a otras en la praxis real del Diseño.

Definir al Diseño como una praxis permite no sólo abordar la referida complejidad, sino también ampliar la perspectiva epistemológica, superando el supuesto antagonismo entre el orden factual y valorativo, desde el momento que integra su comprensión en la unidad de la experiencia, vital y social en que esa práctica se desarrolla ${ }^{7}$.

Entre otras cosas, porque si los valores se fundan en una experiencia vital (=del orden viviente) o social, se puede aceptar que esos valores tienen objetividad: lo que «es» (lo factual) permanece en el ser en tanto ciertos valores se realicen o se mantienen dentro de variaciones que lo hacen sustentable. Lo patológico no es patológico porque una mente externa al «ser» lo juzga como tal, sino porque el propio ser se desrealiza, se empobrece, se deteriora, pierde entidad, si estos valores no se cumplen o no se alcanzan. Y cuando aludimos al «ser» lo hacemos en código filosófico: el concepto se aplica al orden de la vida, como al orden de los objetos construidos.

\section{El Diseño como praxis $^{8}$}

La propuesta de concebir al Diseño como una práctica, y en especial como una práctica social, permite ubicar algunas de las dimensiones que examinamos previamente, conjuntamente con la consideración de sus mutuas y complejas relaciones.

En esa dirección comenzaré por distinguir:

a. Por una parte lo referido al proceso productivo como acto o conjunto de acciones en las que se despliega la actividad de diseñar. Esta perspectiva contempla las condiciones y 
determinaciones objetivas y subjetivas que conducen esa experiencia. Desde los aspectos técnico/instrumentales (aspectos objetivos) hasta los lógicos y cognitivos y creativos (subjetivos).

b. Por la otra, lo referido al producto del Diseño, es decir, al producto /artefacto, contemplando las especificidades del mimo, según las sub-disciplinas del Diseño. En esta dimensión analítica se pueden ubicar aspectos vinculados a la definición epistémico-ontológica del producto, y también a los aspectos semiótico-comunicacionales (incluyendo las cuestiones estético-hermenéuticas) ${ }^{9}$.

Junto a estos aspectos que podríamos concebir como "internos" al quehacer y la práctica del Diseño, se integran otras dos dimensiones referidas a los contextos en las que las mismas se desarrollan. Si los dos criterios previos, refieren a la práctica productiva y el producto en Diseño; estos otros dos remiten a las condiciones y contextos de esa práctica:

c. Por una parte, considera los entornos institucionales inmediatos: es decir, la cuestión que refiere al «desde dónde», "para qué»y «para quién» se diseña. Dichos contextos fijan criterios normativos, valorativos, estéticos, éticos, etc. Son estos contextos los que además consagran tradiciones y referentes, al modo de los paradigmas khunianos (cfr. Iglesias, M.; Bohórquez Nates, et. al., 2013).

d. Por la otra, el contexto social en sentido amplio. Esta dimensión remite a las condiciones sociales en que la práctica del Diseño se realiza. Refiere a las determinaciones de época (modas, estilos, tradiciones culturales), pero también a las condiciones sociales, político, económicas. De modo que estos contextos no sólo fijan las restricciones y posibilidades técno-materiales, sino también simbólicas, valorativas, estéticas desde las que surgen las ideas, los modelos, las necesidades, en una cierta época o contexto social.

En un esquema general, todas las dimensiones referidas podrían presentarse de la siguiente manera ${ }^{10}$ :

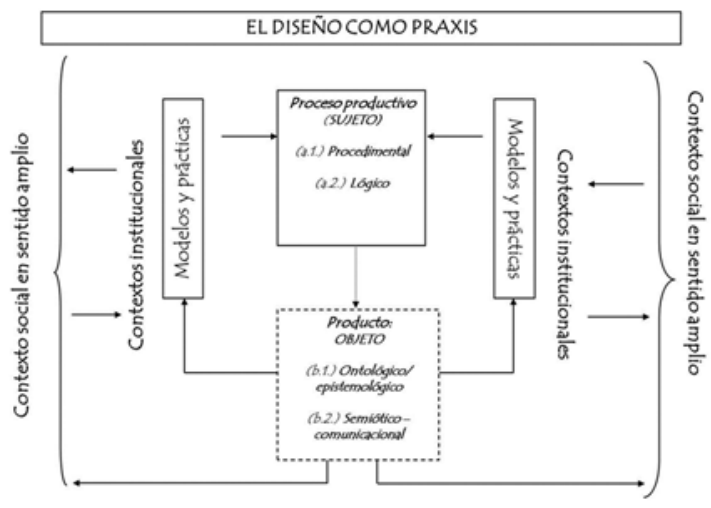


Los cuadrantes centrales refieren al proceso y el producto de Diseño, concebidos desde el interior de la experiencia. Están "rodeados", por así decir, por los contextos institucionales, y por el contexto social en sentido amplio.

Dado el carácter esquemático de la presentación, resulta necesario advertir sobre el alcance y la lectura de la misma. Cada una de las dimensiones reclama a otra en el mismo sentido en que lo hacen los componentes de un nudo borromeo: no se puede considerar a ninguna de ellas sin involucrar a las restantes. El contexto social, por ejemplo, no "rodea" a una práctica, como un elemento exterior o acoplado a ella. El pensamiento del diseñador/a, los componentes y las tradiciones desde las que piensa, imagina, proyecta, están integradas y modeladas por los contextos en que se ha formado, por el ámbito en que trabaja, por el clima de época en que vive. Las técnicas que utiliza, integran tradiciones y valores; comunican y portan efectos simbólicos que pueden escapar incluso a las intenciones deliberadas y conscientes de quién diseña ${ }^{11}$.

Cada uno de los componentes del cuadro, hace posible especificar también el enfoque desde el cual puede abordarse la práctica del Diseño (sin por ello desatender la complejidad de sus mutuas relaciones).

Tentativamente podrían sugerirse las siguientes correspondencias:

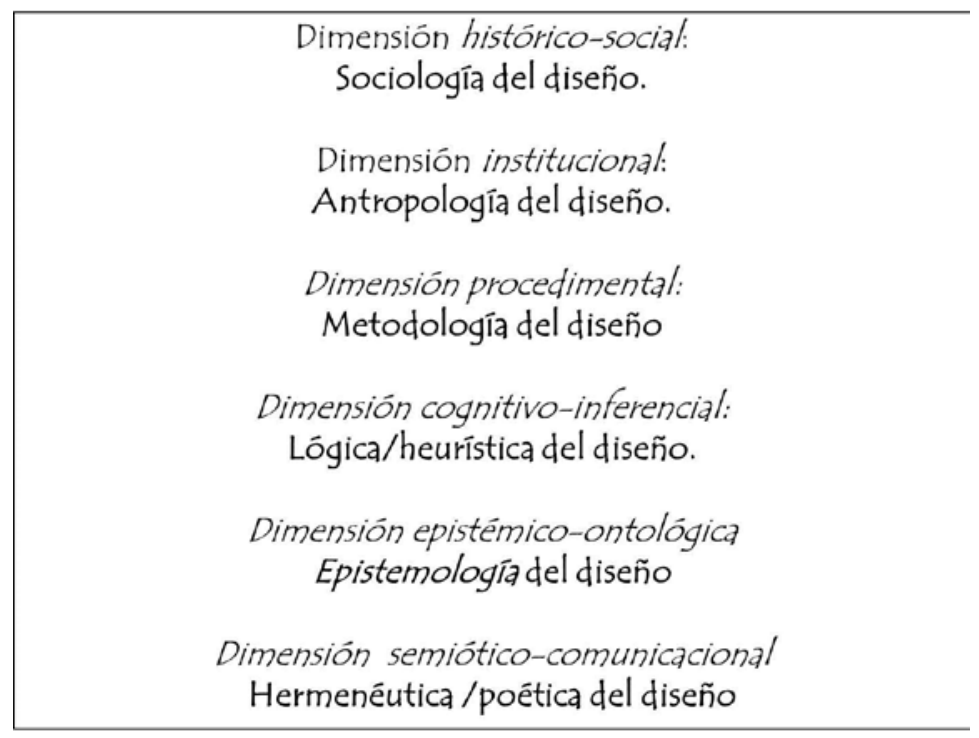

Dado que el tratamiento de cada uno de estos ítems exigiría un desarrollo que excede las posibilidades de este escrito, en lo que sigue se analizará una cuestión que permite, de alguna manera, articularlos a todos ellos en el marco de una reflexión común: la cuestión de la investigación en Diseño. 


\section{El Diseño como medio o como fin de investigación}

Entra las diversas perspectivas en torno al asunto de cómo entender y definir la investigación en Diseño, una propuesta que ha ganado adeptos, es la que se conoce como el paradigma for-trhu-through (Frayling, 1993; Findeli, et.al.; 2008, Herrera Batista, 2010 ${ }^{12}$ ). Con esta definición se alude a la necesidad de distinguir entre la investigación para el Diseño; la investigación sobre el Diseño, y la investigación a través del Diseño ${ }^{13}$.

Se especificará en lo que sigue cada una de esas distinciones, aunque se lo hará con algunos ajustes y revisiones propias.

Se considerará una primera dimensión (no incluida en la referida tríada), definida como la investigación en Diseño. Con ella se alude a la investigación que se sigue -de manera más o menos pautada- en un proceso de Diseño. Corresponde a los tanteos, exploraciones, ensayos que lleva adelante quién diseña, como parte de su rutina de trabajo. Explorar los matices de un color, experimentar con distintas texturas, poner a prueba el efecto de un sonido, pueden concebirse como actividades de investigación. Se testean hipótesis, se explora e incluso se experimenta, pero en el marco de lo que podríamos definir como el en sí de la experiencia. Sin pretensión de validar, ni comunicar dicho proceso investigativo, ya que la "investigación" se realiza de manera muy acotada y directamente vinculada a los fines de la aplicación en el proceso del diseñar.

Un segundo criterio comprendería a la investigación para el Diseño, considerando en este caso a todas aquellas investigaciones que aportan conocimiento aplicable al ámbito del Diseño. Pueden desarrollarse y provenir de otra u otras disciplinas, pero sus resultados tienen interés para el trabajo y la producción en Diseño. Son investigaciones que siguen los métodos propios de la investigación científica (en cualquiera de sus variantes). Ejemplos de este tipo de investigaciones podrían serlo las investigaciones sobre percepción en el campo de la psicología; investigaciones de mercado sobre preferencias e intereses de potenciales destinatarios de un producto, etc. La diferencia entre este tipo de estudios y la investigación en Diseño (que se caracterizó previamente) estriba en que, en estos casos, se siguen procesos metódicamente pautados, mientras que en el caso anterior la investigación es más asistemática e intuitiva. Los resultados de estas investigaciones pueden comunicarse a una comunidad disciplinar, publicarse y difundirse incluso por los medios habituales de la difusión científica.

Tanto el caso de la investigación en Diseño, como para el Diseño, hacen parte de la llamada investigación proyectual, en tanto en ambos casos la investigación es un «medio para»los objetivos de Diseño que se persiguen. Ambas refieren también a los distintos planos en los que se desarrolla la práctica del Diseño: desde el en sí al para sí. En términos de los métodos descriptos por Peirce (1988), esta cuestión podría expresarse en los siguientes términos: el Diseño se sirve y articula el método de la intuición, con el método de la tradición (la imitación e incorporación de los modelos disponibles), con el método de la reflexión (la racionalización de sus procesos) y con el método de la eficacia o de la ciencia (recién este último requiere de la puesta a prueba de los conocimientos del Diseño con métodos controlados y científicos) ${ }^{14}$.

Por su parte, la investigación sobre Diseño, refiere a la investigación que toma por objeto de estudio o bien a la práctica y los procesos productivos en Diseño o bien a los productos del 
Diseño. En este tipo de estudios hay un acercamiento al Diseño algo más externo, ya que en general el interés proviene de otra u otras disciplinas. Ejemplo de este tipo de investigaciones serían aquellas que se interesan por cuestiones sociales, históricas, culturales analizando productos o experiencias de Diseño. También podrían serlo investigaciones destinadas a clasificar o taxonomizar corrientes y estilos en específicos dominios del Diseño.

Finalmente la investigación desde el Diseño, podría concebirse como el caso inverso al de la investigación para el Diseño. Correspondería a investigaciones que producen -desde alguna práctica o producción de Diseño- conocimientos de utilidad para otras disciplinas. Sería el caso, por ejemplo, de "estudios sobre los procesos de creatividad en el contexto de Diseño"; los que podrían resultar de interés al campo de la psicología, o las ciencias cognitivas ${ }^{15}$.

Conforme con estas distinciones, puede reconocerse que la investigación en Diseño tiene muy diversas especificaciones, según sea el enfoque que se privilegie.

De cualquier manera, se torna necesario reconocer también que, no resultan equiparables la metodología del Diseño con la metodología de la investigación científica.

La metodología en Diseño, refiere usualmente (como ya se sugirió) a la que se requiere para la investigación proyectual. Compromete sin duda un trabajo de investigación, pero está absolutamente orientado y circunscripto a resolver el problema fáctico y puntual que el diseñador/a tiene por delante (refiere al orden prescriptivo/valorativo al que aludimos más arriba). Puede usar y servirse de un saber científico, de una técnica (antes que de un método $)^{16}$ de investigación. Pero en todos los casos dicho uso es un medio para resolver la problemática contingente que tiene planteada con su trabajo.

En cambio cuando esa actividad se realiza con los fines propios de la investigación científica, el objetivo de esa experiencia es el de extraer algún aporte o conocimiento de alcance general. Es decir, extraer consecuencias, aprendizajes, teorizaciones que trasciendan al caso puntual para abonar el cuerpo teórico-disciplinar. En estos casos el producto/objeto de Diseño deja de ser el fin último de la investigación, para pasar a ser también un medio, al servicio de la teorización y producción intelectual en la materia.

Es de interés hacer notar que esto mismo ocurre en cualquier práctica o disciplina profesional: se parte de un saber-hacer de primer orden, que está orientada a la intervención o producción en dicha práctica (sea en el campo de las ingenierías, las clínicas médica, etc.). Cuando ese saber-hacer se transforma en objeto de investigación de un nuevo plano analítico, esa práctica (o algún componente de ella) puede transformarse en «objeto de estudio» abordable con los métodos de la investigación científica.

El recorrido de este escrito se ha propuesto situar algunas coordenadas en torno a estos complejos y relevantes asuntos en el campo de la investigación y la producción de conocimiento en las ciencias del Diseño. Se adviertes al finalizar, sin embargo, que esas coordenadas han dejado también un sinnúmero de cuestiones aún abiertas. Se espera que de todos modos, que la reflexión propuesta haya contribuido al menos a seguir precisando interrogantes en este desafiante, y cada vez más consolidado, campo del Diseño. 


\section{Notas}

1. En Iglesias, (2013) se desarrollan y presentan con mucha precisión conceptual las aristas de estos debates en torno a la epistemología en el campo del Diseño.

2. Interesantes consideraciones al respecto pueden encontrarse en el trabajo de Byron Rabé (2014) en el que se sitúan muy distintos enfoques epistemológicos, considerados desde los intereses del Diseño.

3. No se pretende sugerir con esta afirmación que la biología y la sociología se relacionan con sus objetos de la misma manera, ni con los mismos supuestos y métodos de indagación. De hecho la investigación en el campo social ha abierto brechas sustanciales con los supuestos del positivismo clásico. Por ejemplo, las perspectivas discursivistas postulan que en estos dominios el objeto de estudio es siempre una realidad discursiva co-construida entre investigadores/as e investigado/as, a partir de la cual se supera la dicotomía sujeto/ objeto, irreductible desde la perspectiva de las ciencias clásicas. Desde ese marco, se puede afirmar también que la referida realidad discursiva no preexiste a la experiencia de la investigación. Pese a la relevancia de este tema, considero que en lo sustancial, y a los fines de lo que pretendo tematizar aquí, estas distinciones no son relevantes en tanto no modifican los fundamentos de lo que voy a examinar en lo que sigue.

4. Para el desarrollo de este apartado se retoman nociones trabajadas en el texto inédito de la autora: "El Diseño de investigación: entre la táctica y la estrategia" (Ynoub, 2014). De igual modo, se abreva en los aportes de H. Simon, presentados en su obra Las ciencias de lo artificial aunque se plantean varias discrepancias con los postulados que allí se discuten. 5. Nuevamente el referente en esta perspectiva es H. Simon (1979). En su propuesta por hacer científica la formación profesionalista postula la necesidad de formar a los estudiantes en «métodos de optimización» con base en las matemáticas, la estadística y la teoría de la decisión. Bajo el supuesto de que estos métodos de optimización permiten «maximizar el valor esperado de la función de utilidad» estipulada en cada caso.

6. "Estamos acostumbrados a ver y analizar estos objetos desde lo constructivo, lo funcional, lo ergonométrico, lo estético, o lo comunicacional en un esquema que por más que se haya sofisticado últimamente no termina de despegarse de la tríada vitrubiana utilitasvenustas-firmitas" (Bengoa, 2011, p. 6).

7. Dado que no puede desarrollar aquí todas las consecuencias lógico, ontológicos y epistemológicas que se siguen de ello, remito a los lectores al texto de Samaja (2003) "La epistemología de la salud", allí se desarrollan los fundamentos de esta perspectiva en una línea que queda esbozada en la siguiente afirmación: “ ... las normas en el mundo de la vida en los fenómenos prehumanos, son puntos de llegada, y, en cambio, en el mundo de la cultura, son puntos de partida. La vida cotidiana del ser humano sería literalmente impensable si los sujetos no pudiesen, además de distinguir, comunicar la diferencia entre lo que es como debiera ser y lo que debiera ser distinto de lo que es; entre lo correcto y lo incorrecto; entre lo bueno y lo malo; entre el ser y el deber ser" (Samaja, 2003, p. 54).

8. En este apartado se retoman desarrollos del texto de la autora Cuestión de método (Ynoub, 2014). En particular del Cap. II titulado "La ciencia como práctica social”.

9. Se han dado importantes avances que vinculan el campo de la semiótica con el de la actividad proyectual. Esos desarrollos en ocasiones van más allá del aporte de un ins- 
trumento analítico, para constituirse en herramientas categoriales que convergen con los análisis lógico-metodológicos referidos previamente. Entre los trabajos fundacionales en esa línea se cuentan probablemente los de Umberto Eco (1967), de igual modo, Rodriguez et.al. (1973).

10. El esquema que se propone a continuación es una adaptación a los fines de este trabajo, elaborada en base a un esquema análogo presentado en Ynoub (2014, p. 108).

11. Este es el ámbito en el que cobra central relevancia la reflexión y los aportes de la heurística. En lo referido a los factores "ajenos" a las intenciones deliberadas del diseñador/a resultan muy interesantes los desarrollos de Breyer, 2000 sobre lo que define como "Las geometrías del inconsciente".

12. Una clasificación parecida es la que examina Henk Borgdoff (2010) en el "El debate sobre la investigación en las artes" Amsterdam School of the Arts. En este caso el examen se focaliza en el campo del arte, aunque la reflexión y las categorías propuestas se pueden aplicar de igual modo al campo del Diseño.

13. Una reflexión muy próxima a estas ideas aunque tematizada para el campo del arte, puede encontrarse en Azaretto, et. al. (2017).

14. Estos desarrollos se encuentran en un trabajo que tuvo amplia repercusión de Charles Peirce (1988) titulado, "La fijación de las creencias". En nuestro medio Samaja, elaboró una reintepretación de estas nociones que resultarían igualmente aplicables al campo del Diseño (cfr. Samaja, J.; 2003). A la luz de este modelo se puede reconocer que el "método de la reflexión y en especial el método de la eficacia” se inauguran en el Diseño cuando éste se instituye como disciplina, a partir de la escuela Bauhaus y posteriormente con la escuela de Ulm (cfr. Iriarte, I.; Justel, D., 2013).

15. En este grupo podría incluirse un sub-capítulo especificado como investigación a través del Diseño, sería aquella que toma al Diseño como un medio para explorar, investigar, eventualmente enseñar algún otro tema o asunto de interés. Correspondería en el campo del arte, al movimiento denominado Investigación basada en las Artes (IBA) (Arts based Research -ABR- en inglés) el que se inició como parte del giro narrativo (Conelly y Clandinin, 1995, 2000; Lawler, 2002) en la investigación en Ciencias Sociales a principios de los años 80. Vincula, a partir de una doble relación, la investigación con las artes: "Por una parte, desde una instancia epistemológica-metodológica, desde la que se cuestionan las formas hegemónicas de investigación centradas en la aplicación de procedimientos que 'hacen hablar' a la realidad; y por otra, mediante la utilización de procedimientos artísticos (literarios, visuales, performativos, musicales) para dar cuenta de los fenómenos y experiencias a las que se dirige el estudio en cuestión” (cfr. Hernández Hernández, F.; 2008). 16. Un ejemplo en este sentido podrían serlo los desarrollos de Christopher Alexander, que utiliza recursos de las matemáticas para la formalización de fenómenos propios del ámbito de Diseño (cfr. Alexander, 1980). 


\section{Lista de Referencias Bibliográficas}

Alexander (1980). Tres aspectos de matemática y Diseño. Barcelona: Ed. Cuadernos Infimos. Azaretto, et.al. (2017). Investigar en arte. Buenos Aires: Ed. EduLP. Consultado el 27/06/2017 en http://sedici.unlp.edu.ar/bitstream/handle/10915/64154/Documento_completo.pdfPDFA.pdf?sequence $=1$

Bengoa, G. (2011). Visiones del Diseño. Tesis en elaboración. Inédita. Consultada el 27/06/2018 http://www.fadu.edu.uy/sepep/files/2015/06/Curso-7-visiones_G.Bengoa.pdf

Breyer, G. (2000). "Las geometrías del inconsciente". En Bases conceptuales del Diseño. Buenos Aires: Ed. FADU/UBA.

Borgdorff, H. (2010). El debate sobre la investigación en las artes. En Revista de ciencias de la danza, № 13, 2010, págs. 25-46.

Canguilhem, G. (1978). Lo Normal y lo Patológico. México: Ed. S.XXI.

Collingwodd, R. G. (1977). Idea de la historia. México: Ed. Fondo de Cultura Económica.

Conelly, M. y Clandinin, J. (1995). "Relatos de Experiencia e Investigación Narrativa”. En J. Larrosa, et alt. (1995). Déjame que te cuente. Ensayos sobre narrativa y educación. (pp. 11-59). Barcelona: Laertes.

Conelly, M. y Cladinin, J. (2000). Narrative inquiry. San Francisco: Jossey-Bass.

Eco, U. (1967). Appunti per una semiologia delle comunicazioni visive. Milán: Bompanini.

Frayling, C. (1993). Research in art and design. Royal College of Art Research Papers series 1(1). London: Royal College of Art.

Findeli, A. B. (2008). Research Through Design and Transdisciplinarity: A Tentaive Contribution to the Methodology of Design Research.

Hernández Hernández, F. (2008). La investigación basada en las artes. Propuestas para repensar la investigación en educación. En Educatio Siglo XXI, 26 (1) 85-118.

Herrera Batista, M. A. (2010). Investigación y Diseño: reflexiones y consideraciones con respecto al estado de la investigación actual en Diseño. En: No Solo Usabilidad, no 9, 2010.

Iglesias, M. (2013). La epistemología del Diseño como construcción problemática. Anales del IIA (43) 1, 121-134. Consultado el 20/06/2018 en http://www.iaa.fadu.uba.ar/ojs/ index.php/anales/article/viewFile/109/97

Iriarte, I. \& Justel, D. (2013). Considerations in design research. En 17th International Congress on Project Management and Engineering, Logroño, 17-19th July 2013.

Lawler, M. (2002). Narrative in social research. En T. May (Ed.), Qualitative research in action (pp. 242-259). London: Sage.

Peirce, Ch. (1988). La fijación de las creencias. En Ch. S. Peirce El Hombre, un signo. Barcelona: Editorial Crítica.

Rabé, B. (2014). Reflexiones para la epistemología del Diseño. En Revista Avance - Facultad de Arquitectura - Volumen 4- No. 1- 2014.

Rodriguez, J. M. et.al. (1971). Arquitectura como semiótica. Buenos Aires: Ed. Nueva Visión. Samaja, J. (2003). Los caminos del conocimiento. En Semiótica de la Ciencia. Inédito.

Samaja, J. (2003b). Epistemología de la Salud. Buenos Aires: Ed. Lugar.

Simon, H. (1979). La ciencia de lo artificial. Barcelona: Editorial ATE.

Ynoub, R. (2014). Cuestión de método. Aportes para una metodología crítica. México DF: Ed. Cengage Learning. 


\begin{abstract}
The objective of this paper is to contribute some elements of judgement to the debate on the pertinence of epistemology and research methodology in the field of design. It begins by defining the scope of epistemology as a discipline which reflects on the conditions for the production and validation of scientific knowledge. Then, the distinctive traits that knowledge adopts in the field of design are examined, thus arriving at its definition as a knowledge centered on the projection of intended objects. It is proposed that this distinctive trait brings forth issues which have been problematic in classical epistemology, particularly because it relates factual questions ("what something is" or "what something is like") with questions involving values ("what something should be" or "what is desirable"). According to the positivist tradition, modal statements or statements that contain value judgements may not be derived from factual statements. It is proposed, in consequence, that design be examined as a "praxis" (i.e. a social practice) in order to review its epistemological foundations. It is postulated that this perspective overcomes the alleged antagonism between the factual order and that of value judgements, by including in the approach the complexity of the vital and social experience in which the practice of design takes place. From this perspective, the various analytical dimensions with which this complexity may be approached are identified, as well as the fields of academic research and disciplines associated with each of those dimensions. Finally, the consequences of this model for the delimitation of research topics in the field of design are examined.
\end{abstract}

Keywords: epistemology - research methodology - design-praxis.

Resumo: O objetivo deste artigo é fornecer elementos de julgamento para o debate sobre epistemologia e metodologia de pesquisa no campo do design. Nessa direção, começamos por definir o escopo da epistemologia como uma disciplina que trata da reflexão sobre as condições de produção e validação do conhecimento científico. Com base nisso, as características distintivas do conhecimento no campo do design são revisadas, colocando-o como conhecimento do projeto. Propõe-se que estas questões características distintivas de compromisso que têm sido problemático para a epistemologia clássica especialmente porque ligando questões factuais ("o quê") com questões avaliativas ( "o que deveria ser" ou "desejável”). Segundo a tradição positivista, a partir de enunciados factuais não podem ser derivadas declarações modais ou valorativas. Propõe-se, então, assumir o exame do design como uma práxis, ou seja, como prática social, para rever seus fundamentos epistemológicos. Postula-se que esta abordagem supera o suposto antagonismo entre a ordem factual e avaliativo, integrando a abordagem da complexidade da experiência, vida social e que a prática projetual é feito. A partir dessa definição, situam-se as diversas dimensões analíticas com as quais a dita complexidade pode ser abordada, localizando especificidades temáticas e disciplinares para cada uma delas. Por fim, são examinadas as consequências que esse modelo tem para tematizar a pesquisa científica no campo do design.

Palavras-chave: epistemologia - metodologia de pesquisa - design - praxis.

[Las traducciones de los abstracts fueron supervisadas por el autor de cada artículo] 